\title{
Dynamics of the spatial separation of electrons and mobile oxygen vacancies in oxide heterostructures
}

\author{
A. F. Zurhelle $\odot,,^{1,2, *}$ D. V. Christensen $\odot,{ }^{3}$ S. Menzel $\odot,{ }^{2,4, \dagger}$ and F. Gunkel $\odot^{2,3,4, *}$ \\ ${ }^{1}$ Institute of Electronic Materials (IWE 2), RWTH Aachen University, 52074 Aachen, Germany \\ ${ }^{2}$ Jülich Aachen Research Alliance for Fundamentals on Future Information Technology (JARA-FIT), Jülich, Germany \\ ${ }^{3}$ Department of Energy Conversion and Storage, Technical University of Denmark, DK-2800 Kongens Lyngby, Denmark \\ ${ }^{4}$ Peter Grünberg Institute 7, Forschungszentrum Jülich GmbH, 52425 Jülich, Germany
}

(Received 15 April 2020; revised 10 August 2020; accepted 2 September 2020; published 23 October 2020)

\begin{abstract}
In the search for an oxide-based 2D electron system with a large concentration of highly mobile electrons, a promising strategy is to introduce electrons through donor doping while spatially separating electrons and donors to prevent scattering. In $\mathrm{SrTiO}_{3}$, this can be achieved by tailoring the oxygen vacancy profile through reduction, e.g., by creating an interface with an oxygen scavenging layer. Through reduction, oxygen atoms are removed close to the interface, leaving behind oxygen vacancies in the $\mathrm{SrTiO}_{3}$ lattice and mobile electrons in the $\mathrm{SrTiO}_{3}$ conduction band. The commonly assumed picture is that the oxygen vacancies then remain confined close to the interface while the electrons leak a few nanometers into the bulk, resulting in an electron-defect separation and a highly mobile, oxide-based 2D electron system. So far it has remained unclear how the confinement and electron-defect separation develop over time. Here, we present transient finite element simulations that consider three driving forces acting on the oxygen vacancy distribution: diffusion due to the concentration gradient, drift due to the intrinsic electric field, and an oxygen vacancy trapping energy that holds oxygen vacancies at the interface. Our simulations show that at room temperature, three distinct regions are formed in $\mathrm{SrTiO}_{3}$ within days: (1) Oxygen vacancies are partially held at the interface due to the oxygen vacancy trapping energy. (2) The accompanying positive space charge causes an oxygen vacancy depletion layer with large electron concentration and high mobility just below the interface. This electron-defect separation, indeed, leads to a highly conductive region. (3) While we are able to describe measured conductivity data with an oxygen vacancy trapping energy of $-0.2 \mathrm{eV}$, this value does not prevent oxygen vacancy diffusion into the bulk: A diffusion front progresses into the bulk and leads to significant conductivity arising over the first micrometer within a couple of months. An enhanced oxygen vacancy trapping energy of $-0.5 \mathrm{eV}$ or below would suppress this loss of confinement, leading to a static and pronounced electron-defect separation. Consequently, our results highlight the importance of oxygen vacancy redistribution and suggest the trapping energy of oxygen vacancies at the interface as an important design parameter for oxygen-vacancy-based 2D electron systems.
\end{abstract}

DOI: 10.1103/PhysRevMaterials.4.104604

\section{INTRODUCTION}

Oxide heterointerfaces can harbor electronic systems with a variety of remarkable properties, such as field-tunable superconductivity [1-3], magnetic ordering [4-10], or thermoelectricity $[11,12]$, which typically are not observed in the involved undoped bulk compounds. $2 \mathrm{D}$ electron systems at such interfaces offer a fascinating opportunity to study novel physics, which one day may lead to a new generation of oxide electronics [13]. Several reports show 2D electron systems can be tuned through gating [1-2,14-17], growth conditions [18-22], annealing [14,19,22,23], exposure to water [24,25],

\footnotetext{
${ }^{*}$ Corresponding author: a.zurhelle@iwe.rwth-aachen.de

†Corresponding author: st.menzel@fz-juelich.de

¿Corresponding author: f.gunkel@fz-juelich.de
}

Published by the American Physical Society under the terms of the Creative Commons Attribution 4.0 International license. Further distribution of this work must maintain attribution to the author(s) and the published article's title, journal citation, and DOI. and by tuning the interfacial defect structure thermodynamically [9,26-27]. Mainly, two mechanisms are discussed that contribute to the formation of $2 \mathrm{D}$ electron systems at an oxide heterointerface: (a) A polar discontinuity can lead to an electron transfer between the oxides, e.g., at the epitaxial$\mathrm{LaAlO}_{3} / \mathrm{SrTiO}_{3}$ interface [20,27-30]. (b) Oxygen atoms are removed close to the surface or an interface, leaving behind oxygen vacancies, which act as donor-type dopants, and electrons, which then form the 2D electron system [31-33]. This reduction can be achieved by exposure to a reducing atmosphere [34], photon irradiation [35-39], ion bombardment [40], or by growing an oxygen scavenging layer on top $[41,42]$. In the latter case, oxygen atoms are transferred from the oxide to the scavenging layer due to a difference in chemical potential, e.g., at the amorphous- $\mathrm{LaAlO}_{3} / \mathrm{SrTiO}_{3}$ or $\gamma-\mathrm{Al}_{2} \mathrm{O}_{3} / \mathrm{SrTiO}_{3}$ interface $[42,43]$. These mainly oxygenvacancy-based systems potentially reflect a simple way of establishing low-dimensional electron systems, as they can be fabricated at or near room temperature.

A commonly assumed picture is that the oxygen vacancies remain confined to the interface due to either negligible 
diffusion or a lowered formation energy at the interface [41,42,44-47]. The oxygen vacancies act as donor dopants and introduce a large concentration of mobile electrons close to the interface, while the concentration of mobile electrons in the adjacent bulk is very low. The electrons introduced by the oxygen vacancies thus partially diffuse into the bulk. The resulting charge separation gives rise to a positive space charge at the interface and an internal electric field. Consequently, the electrons achieve a dynamic equilibrium between diffusion into the bulk and drift to the interface due to the internal electric field. Hence, the electrons spread from the interface into the first nanometers of the bulk according to their screening length $[42,48,49]$.

Apart from their role as donor-type defects, oxygen vacancies have another effect on the conductivity: As scattering centers for electrons, oxygen vacancies limit the low-temperature mobility. In order to obtain a 2D electron system with high carrier concentration and high mobility, a promising design strategy is to introduce electrons via reduction, but to spatially separate the oxygen vacancies and high-mobility electrons. This strategy of donor-electron separation is known as modulation doping [50,51].

Recently, Schütz et al. found that several months of storage at room temperature lead to a mobility enhancement at $\gamma-\mathrm{Al}_{2} \mathrm{O}_{3} / \mathrm{SrTiO}_{3}$ interfaces, which represent one important $2 \mathrm{D}$ electron system with an oxygen scavenging layer forming oxygen vacancies in $\mathrm{SrTiO}_{3}$ [47]. Supported by density functional theory (DFT) calculations that show a lowered energy for oxygen vacancies at the interface compared to the bulk of $\mathrm{SrTiO}_{3}$, Schütz et al. concluded that oxygen vacancies redistribute to the interface. Because the $2 \mathrm{D}$ electron system extends from the interface up to a few nanometers into the bulk, this is supposed to lead to a separation of charge carriers. They suggest that this increased separation of dopants and electrons reduces the electron scattering and leads to the observed increase in electron mobility.

While there are other studies [19,21-23,31,33,41,50,5254] that report changes in the oxygen vacancy profile at $\mathrm{SrTiO}_{3}$-based heterointerfaces, the involved dynamics of ions in a complex energy landscape, with strong concentration gradients and intrinsic electric fields, has not been discussed in detail yet. Consequently, several questions remain unclear: What are the driving forces involved in the oxygen vacancy redistribution? How are the oxygen vacancies distributed close to the interface? How does the spatial separation of oxygen vacancies and electronic charge carriers develop over time and how confined are these electron systems? Furthermore, other phenomena that emerge at oxide-based 2D electron systems, e.g., magnetism [8] and superconductivity, are expected to be sensitive to the details of the defect profile, and the dynamics of defect redistribution might therefore offer an opportunity to tailor these properties.

To this end, we present a transient 1D finite element model that describes the oxygen vacancy redistribution at $\mathrm{SrTiO}_{3}$-based interfaces. Our simulations show that at room temperature, complex oxygen vacancy profiles develop within days and continue to develop over months; such heterostructures are hence expected to change their properties over time. Using an empirical equation based on electron-defect scattering, we calculate the low-temperature electron mobility from

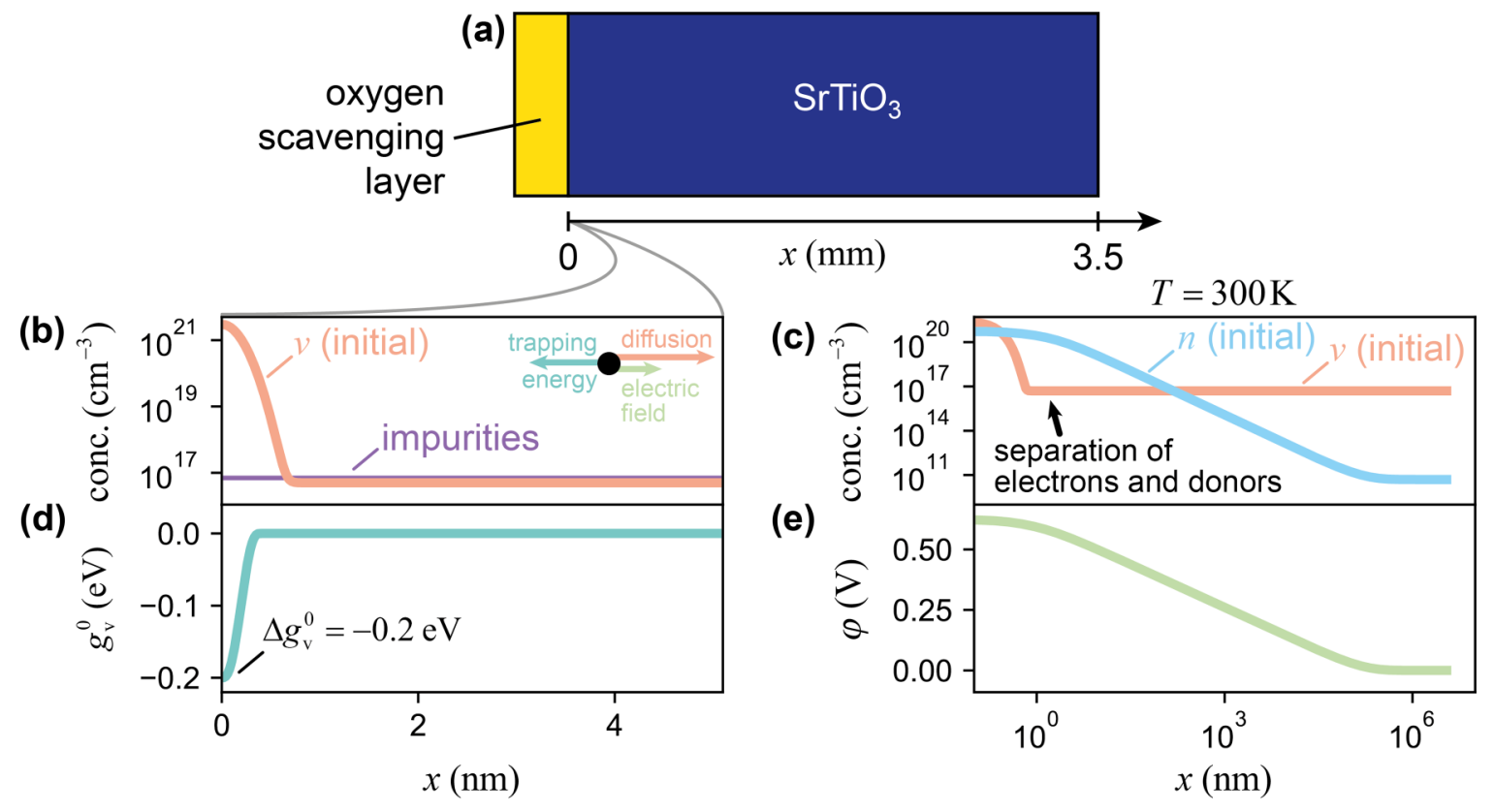

FIG. 1. (a) The assumed geometry is $3.5 \mathrm{~mm}$ of $\mathrm{SrTiO}_{3}$ capped with an oxygen scavenging layer, e.g., $\gamma-\mathrm{Al}_{2} \mathrm{O}_{3}$. (b) The assumed profiles for the initial oxygen vacancy distribution (red) and the background of acceptor-type impurities (purple). The arrows indicate the effect of the three driving forces acting on the oxygen vacancy profile: Due to the oxygen vacancy trapping energy (turquoise arrow), oxygen vacancies are pulled to the interface, while diffusion (red arrow) and electrostatic potential (green arrow) lead to a redistribution into the bulk. (c) The initial oxygen vacancy distribution next to the resulting initial electron profile (blue) at $300 \mathrm{~K}$. The separation of electrons and donors becomes apparent. (d) The profile of the standard chemical potential for oxygen vacancies relative to the bulk. At the interface, this potential is lowered due to the oxygen vacancy trapping energy. (e) The electrostatic potential profile caused by the spatial separation of electrons and donors. The resulting electric field pulls oxygen vacancies into the bulk. 
TABLE I. Values of the parameters used in our simulations. Details on the approximation for the Fermi-Dirac integral $F_{1 / 2}$ in Sec. SM2 and on the lookup table for $\varepsilon_{r}(T, E)$ in Sec. SM3 of the Supplemental Material [79].

\begin{tabular}{lll}
\hline \hline Parameter & \multicolumn{1}{c}{ Value } & \multicolumn{1}{c}{ Comment } \\
\hline$n_{\text {bulk }}$ & $5 \times 10^{10} \mathrm{~cm}^{-3}$ & Bulk electron concentration \\
$N_{\text {impurities }}$ & $10^{17} \mathrm{~cm}^{-3}$ & Background impurity concentration \\
$v_{\text {bulk }}$ & $\frac{1}{2}\left(N_{\text {impurities }}+n_{\text {bulk }}\right)$ & Bulk oxygen vacancy concentration \\
$a$ & $0.3905 \mathrm{~nm}$ & Lattice constant \\
$d_{\text {init }}$ & $0.19525 \mathrm{~nm}$ & $\begin{array}{l}\text { Length scale of initial oxygen vacancy distribution; parameter study } \\
\text { in Sec. SM3 of the Supplemental Material [79] }\end{array}$ \\
$v_{\text {init }}(x)$ & $\left(3 \times 10^{21} \mathrm{~cm}^{-3}-v_{\text {bulk }}\right) \exp \left(-\frac{x^{2}}{d_{\text {init }}^{2}}\right)+v_{\text {bulk }}$ & Initial oxygen vacancy distribution; see Fig. 1(b) \\
$\Delta g_{v}^{0}$ & $-0.2 \mathrm{eV}$ & $\begin{array}{l}\text { Oxygen vacancy trapping energy; parameter study shown in Fig. 4(a) } \\
\text { Standard chemical potential for oxygen vacancies relative to the bulk; }\end{array}$ \\
$g_{v}^{0}(x)$ & $\begin{cases}\Delta g_{v}^{0}\left[-6\left(\frac{x}{a}\right)^{5}+15\left(\frac{x}{a}\right)^{4}-10\left(\frac{x}{a}\right)^{3}+1\right] \\
0 \quad \text { for } x>a\end{cases}$ & for $0 \leqslant x \leqslant a$ Fig. 1(d) \\
$D_{v}(T)$ & $0.33 \times \exp \left(-\frac{\mathrm{eV}}{k_{B} T}\right) \frac{\mathrm{cm}}{\mathrm{s}}$ & Oxygen vacancy diffusion coefficient; from Ref. [68] \\
$N_{C}$ & $4.1 \times 10^{16}\left(\frac{T}{\mathrm{~K}}\right)^{1.5} \mathrm{~cm}^{-3}$ & Effective density of states at the conduction band edge; from Ref. [72] \\
\hline \hline
\end{tabular}

the obtained profiles and see a significant influence of the oxygen vacancy redistribution on the low-temperature mobility with a time evolution agreeing well with experimental data. Finally, we study how an oxygen vacancy trapping energy at the interface affects the confinement and spatial separation of electrons and defects, as this trapping energy will depend on the chosen materials and the interface structure. Our model predicts the local oxygen vacancy and electron distributions, their evolution over time, as well as the expected electron mobility and confinement of the $2 \mathrm{D}$ electron system.

\section{MODEL}

We consider a heterointerface composed of $\mathrm{SrTiO}_{3}$ and an oxygen scavenging layer, which during growth leads to formation of oxygen vacancies and itinerant electrons in $\mathrm{SrTiO}_{3}$. We assign a representative oxygen vacancies profile close to the interface and study the relaxation of this distribution over time under the influence of three driving forces: (1) diffusion due to the oxygen vacancy concentration gradient, (2) drift due to an intrinsic electric field building up through space charge and the associated band bending, and (3) an assumed profile of standard chemical potential for oxygen vacancies, reflecting a trapping potential for oxygen vacancies at the interface.

\section{A. Initial state}

As model system, we assume a 3.5-mm slab of $\mathrm{SrTiO}_{3}$ with an oxygen scavenging layer on the left side [Fig. 1(a)]. The distance from the interface is denoted by $x$. The $\mathrm{SrTiO}_{3}$ slab is slightly acceptor-doped because of inevitable impurities [55-57] (refer to Table I for concentration). These impurities are expected to be homogenously distributed, because they are usually formed during crystal growth and typically have very high migration barriers and low diffusion coefficients $[58,59]$. In the bulk, the negatively charged acceptors are mainly balanced by oxygen vacancies that are doubly positively charged according to the concept of relative charges (see Kröger-Vink notation [60]). The electron concentration is comparably small: We chose a value of $5 \times 10^{10} \mathrm{~cm}^{-3}$ to ensure that the conductivity due to bulk electrons remains negligible throughout our simulations. Note that the exact number only insignificantly influences the described dynamics of the interfacial space-charge layer.

The oxygen scavenging layer, e.g., $\gamma-\mathrm{Al}_{2} \mathrm{O}_{3}[42,47]$, has a lower chemical potential for oxygen. Consequently during growth, oxygen passes over from the $\mathrm{SrTiO}_{3}$ slab to the oxygen scavenging layer, leaving behind a large number of oxygen vacancies and electrons close to the interface. The number of electrons introduced this way can be estimated from measured sheet carrier densities, and is typically found to be around $1 \times 10^{14} \mathrm{~cm}^{-2}$ in high-mobility samples $[42,47,61]$. The number of introduced oxygen vacancies is set to half of this amount, because per oxygen vacancy two electrons are donated to the $\mathrm{SrTiO}_{3}$ conduction band [43].

For the initial oxygen vacancy distribution, we assume that the oxygen vacancies were generated at the interface and have remained nearly completely immobile during sample preparation: the vacancies are spread out on the length scale of half a unit cell [equation given in Table I; profile shown in Fig. 1(b)]. This starting point resembles the simple and often assumed picture that the oxygen vacancies remain confined close to the interface. However, we tested various initial oxygen vacancy distributions and confirmed that they relax to the same complex profile: In a series of simulations, we varied the initial profile depth by spreading out the profiles on length scales of $d_{\text {init }}=0.25$ to 25 unit cells (roughly 0.1 to $10 \mathrm{~nm}$ ), while keeping the total number of oxygen vacancies constant. In each case, the profiles relaxed from the initially chosen distribution to the same profile within a few days (see Sec. SM1 of the Supplemental Material [79]). This ensures that our simulations allow general conclusions on the oxygen vacancy redistribution, although the exact initial distribution is unknown. By choosing this state as a starting point for our simulations, we also assume that the oxygen transfer between the oxygen scavenging layer and the $\mathrm{SrTiO}_{3}$ substrate has already taken place during sample preparation [31,54]. After sample preparation, the rate of oxygen transfer is expected to become insignificant, because the sheet carrier density in such samples typically remains constant over long periods of time [41,47]. 
While the oxygen vacancies are initially confined close to the interface, the electrons have, due to their higher mobility, already achieved a dynamic equilibrium between drift and diffusion [see Fig. 1(c)]: The electron concentration gradient leads to electrons diffusing into the bulk. The resulting charge separation leads to a positive space charge at the interface (due to the oxygen vacancies) and an internal electric field, which pulls the electrons to the interface. As a result, the electron profile reaches further into the bulk than the oxygen vacancy profile due to the screening length of the electrons. This separation of electrons and donors (oxygen vacancies) is often assumed to result in a layer of large electron concentration with high mobility.

\section{B. Electron profile and electrostatic potential}

From the distribution of oxygen vacancies, the electron profile and the electrostatic potential are calculated with Poisson's equation. Together with the background concentration of acceptor-type impurities $N_{\text {impurities }}$, the (in Kröger-Vink notation [60]) doubly positively charged oxygen vacancies $v$ lead to an effective dopant concentration $N_{D}^{+}[45,46]$ :

$$
N_{D}^{+}(x)=2 v(x)-N_{\text {impurities }} .
$$

From this, the electrostatic potential $\varphi$ and the electron concentration $n$ can be obtained by solving Poisson's equation

$$
\begin{aligned}
-\frac{d}{d x}\left[\varepsilon_{0} \varepsilon_{r}(E, T) \frac{d \varphi}{d x}\right] & =e\left[N_{D}^{+}(x)-n(x)\right] \\
& =e\left[N_{D}^{+}(x)-N_{C} F_{1 / 2}(\eta(x))\right],
\end{aligned}
$$

with

$$
\eta(x)=\eta_{\mathrm{ref}}+\frac{e}{k_{B} T} \varphi(x) .
$$

Here, $\varepsilon_{0}$ is the vacuum permittivity, $\varepsilon_{r}$ is the relative permittivity (dependant on electric field $E$ and temperature $T$ ), $k_{B}$ is the Boltzmann constant, $e$ is the elementary charge, $N_{C}$ is the effective density of states at the conduction band, $F_{1 / 2}$ is the Fermi-Dirac integral, and $\eta$ denotes the energy difference between Fermi level and conduction band edge in units of $k_{B} T$. The energy difference $\eta_{\text {ref }}$ is determined by imposing the constraint that global electroneutrality has to be fulfilled. The Fermi-Dirac integral $F_{1 / 2}$ was approximated using an approach by Bednarczyk et al. [62] (details in Sec. SM2 of the Supplemental Material [79]).

Electric fields can significantly reduce the permittivity at $\mathrm{SrTiO}_{3}$-based heterointerfaces $[63,64]$. Thus, we used a numerical model by Hemberger et al. [65], which allows us to treat the field and temperature dependence of the relative permittivity of $\mathrm{SrTiO}_{3}$. With this model, we generated a look-up table for $\varepsilon_{r}$ as function of temperature and electric field, which was interpolated during the simulations (details in Sec. SM2 of the Supplemental Material [79]).

As boundary conditions for the electrostatic potential, we assume a zero-flux Neumann boundary condition at the interface $(x=0 \mathrm{~nm})$, i.e., negligible electric field directly at the interface. This is expected for oxide heterostructures, where the electrons are mainly introduced through oxygen transfer, not charge transfer [43]. At $x=3.5 \mathrm{~mm}$, the potential is chosen to be zero (Dirichlet boundary condition). The resulting initial potential profile evaluated at $T=300 \mathrm{~K}$ is shown in Fig. 1(e).

\section{Dynamics of the oxygen vacancy redistribution}

The dynamics of the system are addressed by describing how the oxygen vacancy profile evolves over time. That is, we solve the continuity equation for the oxygen vacancies,

$$
\frac{d v}{d t}=-\frac{d j_{v}}{d x}
$$

with the current density of oxygen vacancies $j_{v}$ and zero-flux Neumann boundary conditions for both boundaries; i.e., no oxygen vacancies are exchanged with the surroundings or the oxygen scavenging layer. This is consistent with the observation that the sheet carrier density in such samples remains constant over long periods of time at room temperature [47]. Based on the approach that the general cause of an ionic current is a gradient in electrochemical potential [66], we assume the following equation for the current density of oxygen vacancies:

$$
j_{v}(x)=-\frac{D_{v}}{k_{B} T}\left(k_{B} T \frac{d v}{d x}+2 e v(x) \frac{d \varphi}{d x}+v(x) \frac{d g_{v}^{0}}{d x}\right),
$$

where $D_{v}$ is the diffusion coefficient of oxygen vacancies. For $\mathrm{SrTiO}_{3}$, this diffusion coefficient has been investigated extensively and measured at temperatures as low as room temperature [67-71] (see Table I for the relation between the diffusion coefficient and temperature used in this work).

The above equation considers three driving forces for the oxygen vacancy redistribution [also indicated by the colored arrows in Fig. 1(b)]: (1) Due to the initial concentration gradient $(d v / d x)$, oxygen vacancies diffuse away from the interface into the bulk (red arrow) as described by Fick's first law. (2) The intrinsic electric field $(-d \varphi / d x)$ mainly pulls oxygen vacancies away from the interface (green arrow). (3) Oxygen vacancies have a lower Gibbs energy of formation at the interface than in the bulk; i.e., the difference in the standard chemical potential $\Delta g_{v}^{0}$ for oxygen vacancies at the interface relative to the bulk is negative (as described in the literature [45-47]). $\Delta g_{v}^{0}$ causes a gradient in the standard chemical potential $\left(d g_{v}^{0} / d x\right)$ that pulls oxygen vacancies to the interface and traps them there (turquoise arrow). Thus, we refer to $\Delta g_{v}^{0}$ as oxygen vacancy trapping energy [Fig. 1(d)].

How large the oxygen vacancy trapping energy is depends on the nature of the heterostructure and the choice of materials. For now, we assume a value of $-0.2 \mathrm{eV}$, which has been predicted for the $\gamma-\mathrm{Al}_{2} \mathrm{O}_{3} / \mathrm{SrTiO}_{3}$ interface with DFT calculations [47]. In Sec. IIID and Fig. 4, we show a study in which we varied this parameter, to discuss the influence of this thermodynamic driving force.

The assumed profile of the standard chemical potential $g_{v}^{0}$ for oxygen vacancies relative to the bulk is shown in Fig. 1(d). At the interface, $g_{v}^{0}$ starts at $\Delta g_{v}^{0}=-0.2 \mathrm{eV}$ and increases to zero over the first unit cell. The confinement of this change in $g_{v}^{0}$ is based on the assumption that the Gibbs energy of formation for the oxygen vacancy is determined mainly by 
next-neighbor interactions [46]. This assumption is in agreement with DFT calculations [47]. In order to obtain a smooth derivative $d g_{v}^{0} / d x$ [see Eq. (5)], we chose to model $g_{v}^{0}$ as a polynomial in the first unit cell with a derivative of zero at the unit cell boundaries (see Table I).

The continuity equation [Eq. (4)] is solved in 1D using the backward differentiation formula implemented in COMSOL Multiphysics. Because electrons have relaxation times many orders of magnitude shorter than the simulated time steps, the electrons are assumed to adapt to the oxygen vacancy redistribution and achieve a new drift-diffusion balance instantaneously (dynamic equilibrium). Thus, the electrostatic potential and electron profile are calculated by solving Poisson's equation together with the continuity equation of oxygen vacancies. The parameters are listed in Table I.

\section{RESULTS}

The initial state of our simulation resembles the often assumed picture of oxygen-vacancy-based 2D electron systems: the oxygen vacancies are confined to the interface region, where they cause a positive space charge, while the electrons are spread slightly further into the bulk. As shown in Fig. 1(c), the electron concentration is around $5 \times 10^{20} \mathrm{~cm}^{-3}$ at the interface and decreases by 10 orders of magnitude, reaching the bulk concentration after a couple of hundred micrometers. On the first $4 \mathrm{~nm}$, the electron concentration drops by one magnitude and, thus, the electron system can be viewed as confined on the nanometer scale. Over course of time, however, the (slow) motion of ionic defects in response to the concentration gradient, electric field distribution, and energy landscape leads to a departure from this simple depth profile.

\section{A. Simulated oxygen vacancy redistribution}

As shown in Fig. 2(a), even at room temperature, where ionic motion is typically sluggish, a complex depth profile is established on the timescale of a couple of hours to days. The profiles show three features: (1) Oxygen vacancies accumulate close to the interface, within the first nanometer, due to the oxygen vacancy trapping energy. (2) An oxygen vacancy depletion layer forms on the next couple of nanometers. Here, the standard chemical potential for oxygen vacancies $g_{v}^{0}$ has returned to its bulk value, while the positive space charge at the interface pushes the oxygen vacancies deeper into the bulk. (3) A diffusion front of oxygen vacancies has progressed further into the bulk. This redistribution is driven by the concentration gradient of the oxygen vacancies and by the internal electric field (ambipolar diffusion). The oxygen vacancy accumulation (feature 1) and the accompanying depletion layer (feature 2) are caused by the oxygen vacancy trapping energy and the resulting force acting on the oxygen vacancies. Without this force that holds the vacancies at the interface, the oxygen vacancies spread from the interface into the bulk and the profiles only show the ambipolar diffusion front (feature 3 ). Simulated profiles without an oxygen vacancy trapping energy are shown in Sec. SM4 of the Supplemental Material [79].

After 14 days, the oxygen vacancy concentration at the interface is around $10^{21} \mathrm{~cm}^{-3}$. As shown in Fig. 2(a), the accompanying depletion layer extends from approximately 1 $\mathrm{nm}$ to $10 \mathrm{~nm}\left(v \approx 1 \times 10^{18} \mathrm{~cm}^{-3}\right)$, and the diffusion front has reached a depth of about $100 \mathrm{~nm}\left(v \approx 6 \times 10^{18} \mathrm{~cm}^{-3}\right)$. The electrons are only held close to the interface by the positive (a)

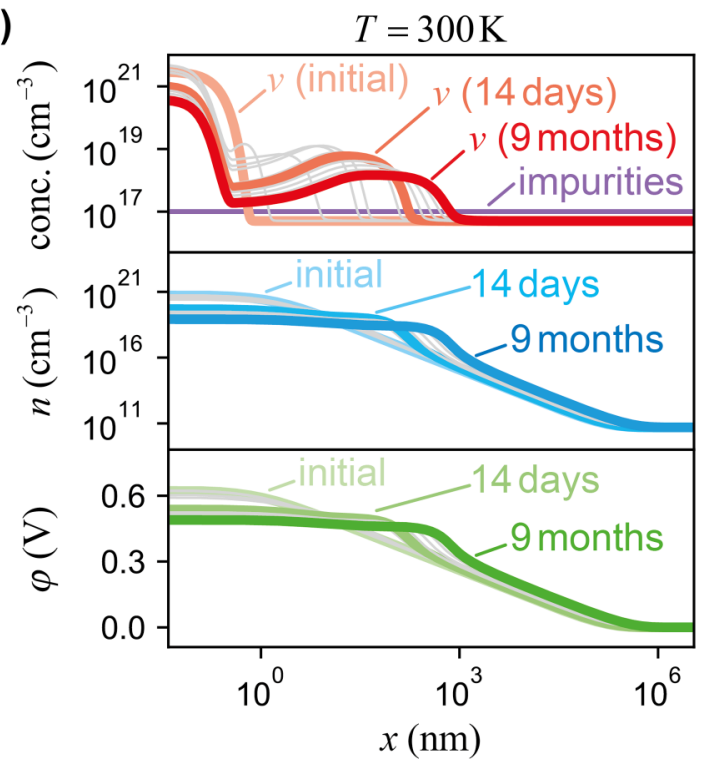

(b)

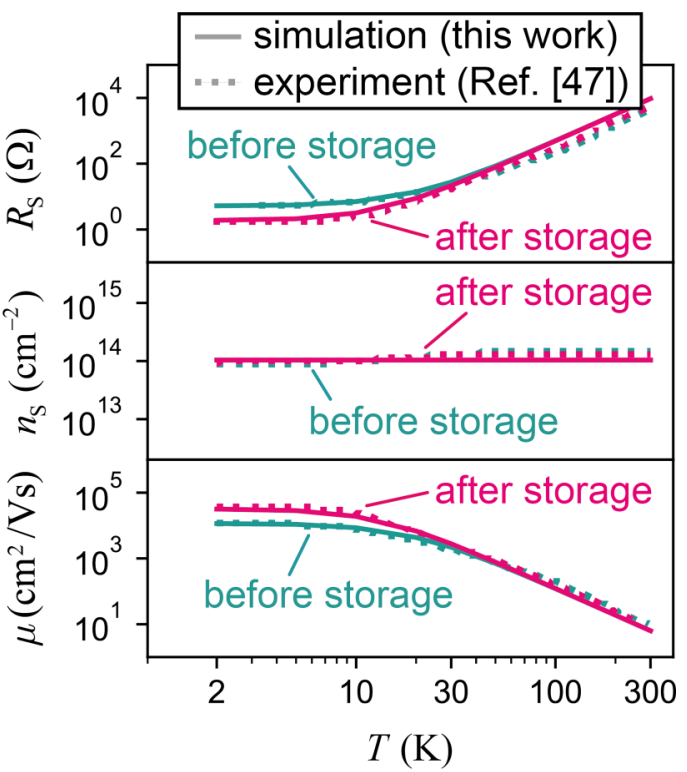

FIG. 2. Simulated redistribution of oxygen vacancies at room temperature for an oxygen vacancy trapping energy of $\Delta g_{v}^{0}=-0.2 \mathrm{eV}$. (a) Development of oxygen vacancy concentration $v$ (red), electron concentration $n$ (blue), and the electrostatic potential $\varphi$ (green) over 9 months of storage at room temperature. The initial profile as well as the profiles after 14 days and 9 months are colored in a light, medium, and darker shade, respectively. Profiles of intermediate times are shown in gray. The assumed concentration of acceptor-type impurities is included for reference (purple). (b) Dotted lines: Measured temperature dependence of sheet resistance $R_{S}$, sheet carrier density $n_{S}$, and mobility $\mu$ before (green) and after (red) several months of storage at room temperature [47]. Solid lines: Comparable data calculated from the simulated concentration profiles after 14 days and 9 months. The change of the low-temperature mobility with time is shown in Fig. 3(a). 
space charge due to the accumulated interfacial oxygen vacancies. Thus, with the oxygen vacancies diffusing into the bulk, the electrons also redistribute: At $300 \mathrm{~K}$, their concentration at the interface is around $5 \times 10^{19} \mathrm{~cm}^{-3}$. The oxygen vacancy diffusion front gives rise to a shoulder in the electron profile: the concentration only drops to $1 \times 10^{19} \mathrm{~cm}^{-3}$ at $100 \mathrm{~nm}$. It is noteworthy that while the oxygen vacancies show depletion in the region from $1 \mathrm{~nm}$ to $10 \mathrm{~nm}$, the electron concentration is still quite large in this region. The lowered concentration of scattering centers, here, causes a layer of electrons with high mobility at low temperatures.

After 9 months, the oxygen vacancy concentration close to the interface has dropped further and reached a value of $4 \times 10^{20} \mathrm{~cm}^{-3}$. The depletion layer has slightly extended (to $15 \mathrm{~nm}$ ) and now reaches concentrations as low as $3 \times$ $10^{17} \mathrm{~cm}^{-3}$. The diffusion front has progressed to a depth of $500 \mathrm{~nm}\left(v \approx 1 \times 10^{18} \mathrm{~cm}^{-3}\right)$. The electron concentration at $300 \mathrm{~K}$ has dropped to $9 \times 10^{18} \mathrm{~cm}^{-3}$ on the first nanometer and $3 \times 10^{18} \mathrm{~cm}^{-3}$ at a depth of $500 \mathrm{~nm}$.

\section{B. Comparison to conductivity measurements}

In order to compare our simulation results to the experimental data in Fig. 2(b), we extracted the sheet conductivity and mobility as would be measured in temperature-dependent conductivity experiments from our calculations. With the oxygen vacancy profile at a certain time, e.g., 14 days, Poisson's equation was solved for several temperatures. This yields the electron profiles for each of these temperatures. The local electron mobility $\mu_{\text {local }}$ was then calculated as function of temperature and local density of scattering centers $N_{\text {scatter }}$ based on the empirical relation found by Moos and Härdtl [73-76],

$$
\mu_{\text {local }}(x)=\frac{2.5 \times 10^{22}}{8.1 \times 10^{14}(T / \mathrm{K})^{2.7}+N_{\text {scatter }}(x) / \mathrm{cm}^{-3}} \frac{\mathrm{cm}^{2}}{\mathrm{~V} \mathrm{~s}},
$$

with $N_{\text {scatter }}(x)=v(x)+N_{\text {impurities }}$; i.e., oxygen vacancies and acceptor-type impurities are treated as scattering centers for electrons. While the scattering on impurities and oxygen vacancies limits the mobility at low temperatures it becomes insignificant at higher temperatures where other scattering mechanisms dominate [61,77]. In Eq. (6), this dependence is phenomenologically described by the power law.

We define the average electron mobility $\mu$ as the ratio of sheet conductance to sheet carrier density obtained by integrating the local conductivity and carrier density over the entire $\mathrm{SrTiO}_{3}$ slab thickness:

$$
\mu=\frac{\int \sigma(x) d x}{e \int n(x) d x}=\frac{\int \mu_{\text {local }}(x) n(x) d x}{n_{S}} .
$$

Schütz et al. measured the temperature dependence of the sheet resistance $R_{S}$, sheet carrier density $n_{S}$, and mobility $\mu$ for $\gamma-\mathrm{Al}_{2} \mathrm{O}_{3} / \mathrm{SrTiO}_{3}$ samples before and after a storage period (several months at room temperature) [47]. Figure 2(b) shows that the data extracted from the simulation results are in good agreement with the measured data, indicating that the change in electrical properties observed during storage can be explained consistently by the redistribution of oxygen vacancies in the vicinity of the interface.

\section{Impact of oxygen vacancy redistribution on the low-temperature mobility}

The simulated profiles allow a detailed analysis of how the oxygen vacancy redistribution at room temperature influences the low-temperature mobility. Our simulations show an increase in the averaged electron mobility $\mu$ at $2 \mathrm{~K}$ upon room temperature storage on a timescale of several months. This mobility enhancement has previously been observed in measurements by Schütz et al. [47] after several months of storage in a vacuum desiccator. In Fig. 3(a), the two measured lowtemperature mobilities-determined 13 days and 8 months after deposition-are compared to the simulated development of the low-temperature mobility. The data extracted from our simulation show excellent agreement with the measured data, considering that the parameters were not further optimized to fit the measurements (see Table I).

Based on our simulations, the origin of the mobility enhancement can now be understood in greater detail: With Fig. 3(b), we will now discuss how much each region contributes to the sheet conductance and how this develops over time. This is closely related to the confinement of the electron system, which is especially important for designing devices utilizing the novel and versatile physics that arise at these heterointerfaces.

At low temperatures, the profiles of oxygen vacancies and background impurities determine the local mobility [Fig. 3(b), top]. In the bulk, these concentrations are given by $N_{\text {impurities }} \approx$ $2 v_{\text {bulk }}$ and, according to Eq. (6), the electron mobility then amounts to $1.6 \times 10^{5} \mathrm{~cm}^{2} / \mathrm{V} \mathrm{s}$ (at $T=2 \mathrm{~K}$ ). In the local mobility profile [Fig. 3(b), second from top], this is also the maximum for the electron mobility, because the additional oxygen vacancies that were introduced at the interface only reduce the mobility. Thus, the local mobility is lowest close to the interface and shows a local maximum in the oxygen vacancy depletion layer.

The product of local mobility and electron concentration yields the local conductivity [Fig. 3(b), third from top]. Because of the low electron concentration in the bulk, the conductivity there is negligible. In the oxygen vacancy depletion layer, however, the enhanced mobility and the large electron concentration give rise to a highly conductive region close to the interface. In addition, the region up to and slightly beyond the diffusion front shows significant conductivity.

Throughout the period from 14 days to 9 months, the local conductivity on the first $150 \mathrm{~nm}$ remains nearly unchanged: While the vacancy concentration in this region decreases with time increasing the mobility, also the electron concentration drops lowering the number of charge carriers. However in the region from $150 \mathrm{~nm}$ to around $1.5 \mu \mathrm{m}$, the progress of the diffusion front during the storage time leads to a significant increase in conductivity.

The integral of the local conductivity plotted over the distance from the interface $x$ [Fig. 3(b), bottom] illustrates how the total conductance is composed: The profiles at 14 days result in a total conductance of around $10 \mathrm{~S}(T=2 \mathrm{~K})$. The highly conductive region in the oxygen depletion layer yields $3 \mathrm{~S}$ and the region up to the diffusion front adds another $7 \mathrm{~S}$. After 9 months, the first $150 \mathrm{~nm}$ still contribute around $10 \mathrm{~S}$, but now the total conductance amounts to around $30 \mathrm{~S}$. The 
(a)

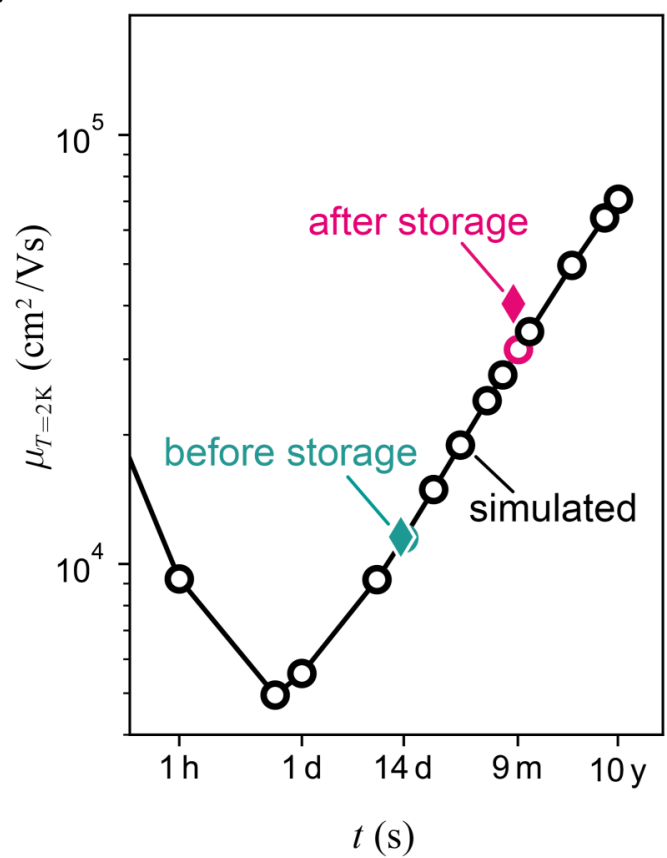

(b)

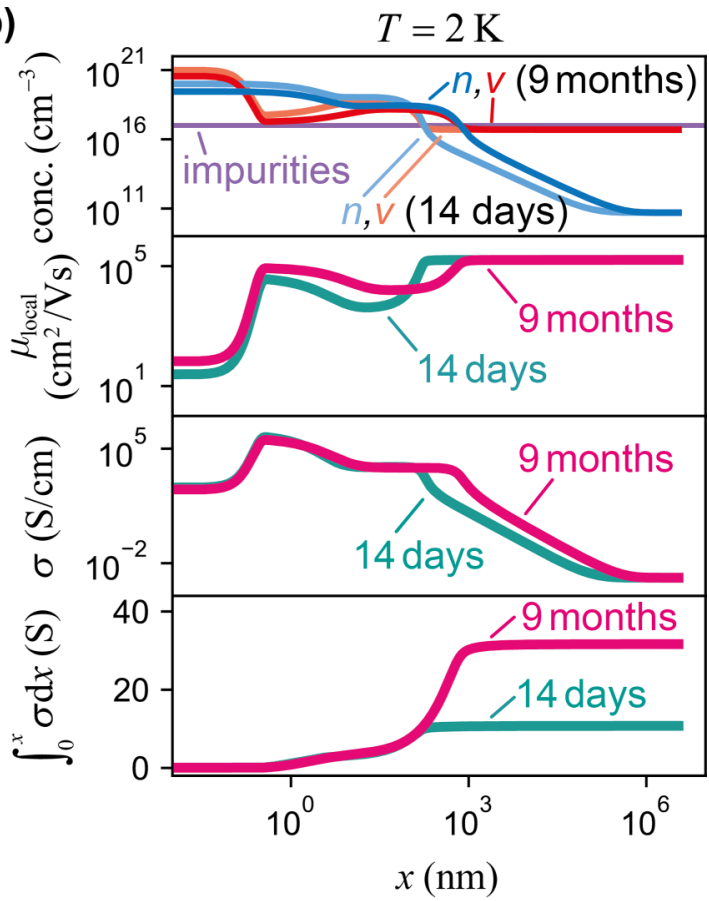

FIG. 3. Effect of oxygen vacancy redistribution on the low-temperature mobility, assuming an oxygen vacancy trapping energy of $\Delta g_{v}^{0}=$ $-0.2 \mathrm{eV}$. (a) Development of the mobility at $2 \mathrm{~K}$ calculated from the simulated concentration profiles (circles) next to measured data (diamonds) from Ref. [47]. The measurements were taken 13 days (green) and 8 months (red) after deposition. The data points from simulations for 14 days and 9 months that are compared to the measurements are colored accordingly. (b) Top: Low-temperature ( $2 \mathrm{~K}$ ) concentration profiles of oxygen vacancies $v$ and electrons $n$ after 14 days (lighter color) and 9 months (darker color) of storage at room temperature. These concentrations determine the local mobility $\mu$ (second plot from top) and the local conductivity $\sigma$ (third plot from top). Bottom: The integral of the local conductivity (conductance up to $x$ ) is plotted as a function of $x$.

enhanced conduction mainly originates from the progress of the oxygen vacancy diffusion front deeper into the bulk. Consequently, our model indicates that electrical measurements of such samples might be strongly influenced by the progress of oxygen vacancy diffusion into the bulk and thus by the exact sample history. For designing devices, this means the electron system loses confinement on the scale of several hundred nanometers; about two-thirds of the conductance does not originate close to the interface and, thus, will likely not be influenced by the physics at the heterointerface.

\section{Influence of the oxygen scavenging layer}

In this model, the influence of the oxygen scavenging layer is (1) to form the initial oxygen vacancy distribution, (2) to serve as a blocking layer preventing diffusion of oxygen vacancies across the interface, and (3) to provide an interface that restrains the diffusion of oxygen vacancies into the bulk due to the trapping energy $\Delta g_{v}^{0}$. A different choice for oxygen scavenging layer or a modification of the interface will likely result in a different value for $\Delta g_{v}^{0}$. In our simulations, we are able to tune this parameter to study its influence on the low-temperature mobility and oxygen vacancy redistribution.

Figure 4(a) shows the development of the low-temperature mobility over time for oxygen vacancy trapping energies in the range of 0 to $-1.4 \mathrm{eV}$. For weak oxygen vacancy trapping $\left(\Delta g_{v}^{0} \geqslant-0.2 \mathrm{eV}\right)$, the simulations show an enhancement of low-temperature mobility over months and years. This can be attributed to the diffusion of oxygen vacancies into the bulk as discussed in the previous section (see Sec. SM4 of the Supplemental Material [79] for profiles with $\Delta g_{v}^{0}=0 \mathrm{eV}$ ). Consequently, oxygen vacancy trapping energies in this range agree well with the electrical measurements by Schütz et al. [47].

By contrast, strong oxygen vacancy trapping $\left(\Delta g_{v}^{0} \leqslant\right.$ $-0.5 \mathrm{eV}$ ) causes a mobility enhancement within a couple of days that then persists over years. As can be seen in Fig. 4(b), the force caused by $\Delta g_{v}^{0}=-0.5 \mathrm{eV}$ becomes strong enough to largely suppress the diffusion of oxygen vacancies into the bulk; instead the oxygen vacancies are mainly pulled to the interface and trapped there. An oxygen vacancy depletion layer develops close to the interface within days and remains stable over several years.

The effect of this oxygen vacancy distribution on the low-temperature properties of the heterostructure is shown in Fig. 4(c). On first sight, a local minimum in the electron profiles at low temperatures becomes apparent. It results from the defect distribution and the reduced screening length at low temperatures. A detailed discussion can be found in Sec. SM5 of the Supplemental Material [79].

In the oxygen vacancy depletion layer, the oxygen vacancy concentration drops far below the bulk value and the local electron mobility approaches its upper limit $2.4 \times$ $10^{5} \mathrm{~cm}^{2} / \mathrm{V} \mathrm{s}$ (at $T=2 \mathrm{~K}$ ) that is determined by the background concentration of impurities. Together with the large electron concentration in this region, this results in a highly conductive layer close to the interface. While there is an oxygen vacancy diffusion front that progresses into the bulk, 
(a)

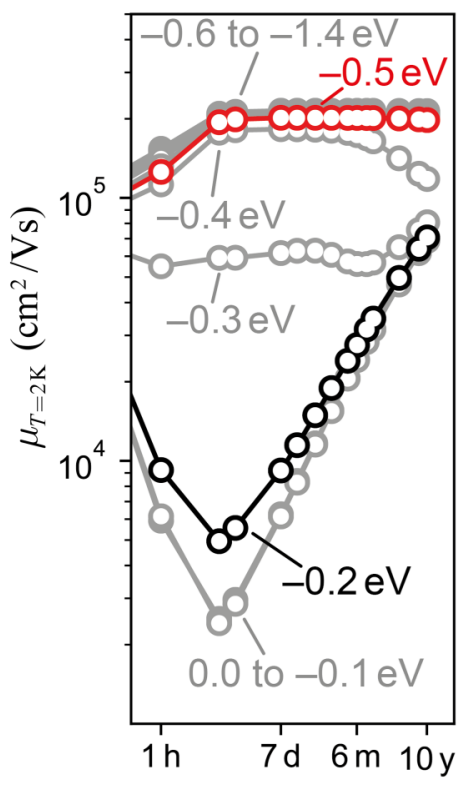

$t(\mathrm{~s})$ (b) $\Delta g_{\mathrm{v}}^{0}=-0.5 \mathrm{eV}, T=300 \mathrm{~K}$

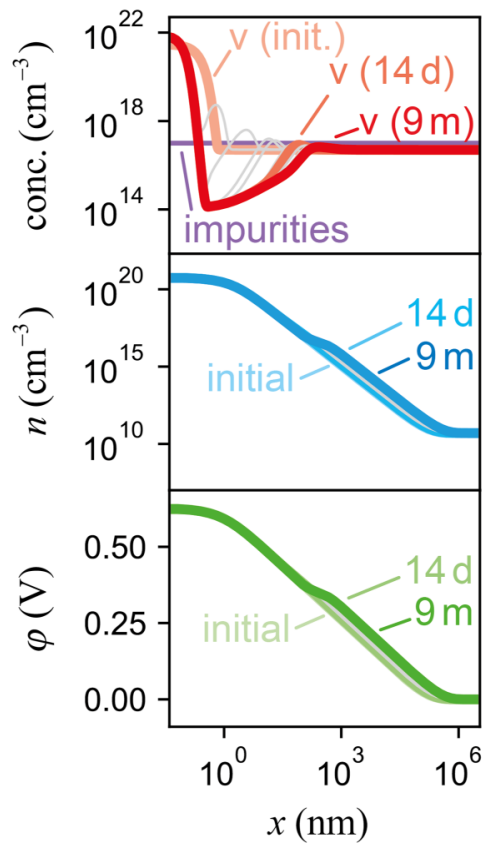

(c) $\Delta g_{\mathrm{v}}^{0}=-0.5 \mathrm{eV}, T=2 \mathrm{~K}$

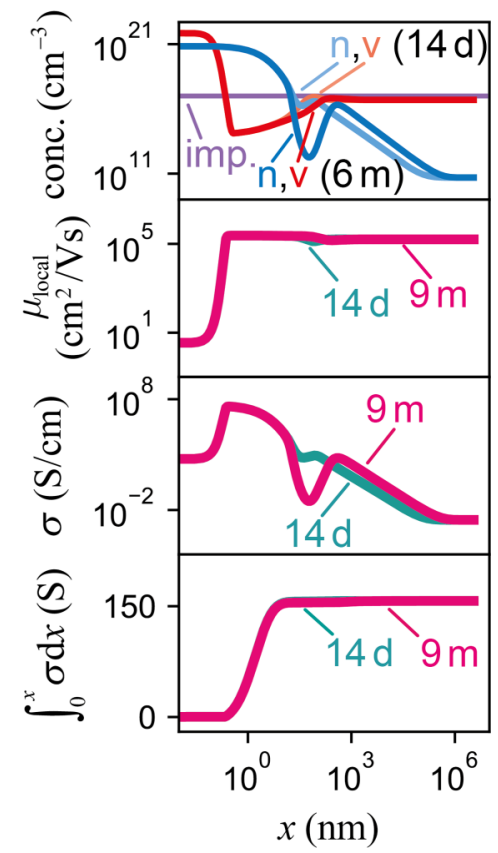

FIG. 4. (a) Influence of the oxygen vacancy trapping energy $\Delta g_{v}^{0}$ on the development of the low-temperature mobility. (b) For $\Delta g_{v}^{0}=$ $-0.5 \mathrm{eV}, T=300 \mathrm{~K}$ : Development of oxygen vacancy concentration $v$ (red), electron concentration $n$ (blue), and the electrostatic potential $\varphi$ (green) over 9 months of storage at room temperature. The assumed concentration of acceptor-type impurities is included for reference (purple). (c) For $\Delta g_{v}^{0}=-0.5 \mathrm{eV}, T=2 \mathrm{~K}$ : Low-temperature concentration profiles of oxygen vacancies $v$ and electrons $n$ after 14 days (lighter color) and 9 months (darker color) of storage at room temperature. These concentrations (top) determine the local mobility $\mu$ (second from top) and local conductivity $\sigma$ (third from top). Bottom: The integral of the local conductivity (conductance up to $x$ ) is plotted as a function of $x$.

it has no significant effect on the conductance. Because the mobility becomes mostly independent of the oxygen vacancy profile, further lowering the oxygen vacancy trapping energy below $\Delta g_{v}^{0}=-0.5 \mathrm{eV}$ only insignificantly influences the low-temperature mobility [cf. Fig. 4(a)].

This electron system only shows significant conductivity close to the interface; i.e., it is confined and its conductance will likely be strongly influenced by the physics of the heterointerface. The stable confinement is achieved within days and remains stable over years. Furthermore, the lowtemperature mobility reaches close to the upper limit given by the background concentration of impurities. This suggests that $\mathrm{SrTiO}_{3}$-based heterointerfaces can be optimized by finding a system with an oxygen vacancy trapping energy below $\Delta g_{v}^{0}=-0.5 \mathrm{eV}$. More generally, searching for oxygen-vacancy-based 2D electron systems with a large oxygen vacancy trapping energy and a low impurity concentration might be a promising approach to finding a strongly confined, highly conductive 2D electron system.

\section{CONCLUSIONS}

We have presented simulations of the oxygen vacancy redistribution at oxygen-vacancy-based heterointerfaces, considering three driving forces acting on the oxygen vacancy profile: diffusion due to a concentration gradient, drift due to an internal electric field, and the effect of an oxygen vacancy trapping energy $\Delta g_{v}^{0}$ at the interface. We show that the redistribution of oxygen vacancies causes complex concentration profiles to develop at room temperature within days and to continue to develop over years. Oxide heterostructures with conductivity arising from oxygen vacancies are hence expected to change their properties over time.

With an oxygen vacancy trapping energy $\Delta g_{v}^{0}=-0.2 \mathrm{eV}$, we were able to describe experimental conductivity data [47] of a $\gamma-\mathrm{Al}_{2} \mathrm{O}_{3} / \mathrm{SrTiO}_{3}$ system and gain insight into the oxygen vacancy redistribution: The oxygen vacancies and electrons introduced at the interface diffuse into the bulk (ambipolar diffusion). The oxygen vacancy trapping energy causes an accumulation of oxygen vacancies at the interface, which is accompanied by an oxygen vacancy depletion layer on the length scale of several nanometers. This results in a highly conductive region close to the interface with a width of a few nanometers. From the depletion layer to a little further than the diffusion front, a region of enhanced conductivity is established that significantly contributes to the conductance. This region broadens with the progress of the diffusion front. This loss in confinement leads to an enhanced low-temperature conductance after several months at room temperature, which agrees with previous measurements [47]. Consequently, the oxygen vacancy profile can be expected to strongly depend on the exact sample history, and this can impact all properties of $2 \mathrm{D}$ electron systems that are sensitive to the oxygen vacancy distribution or more generally the confinement, e.g., magnetism [8] or magnetoresistivity [78].

In our simulations, we found that lowering the oxygen vacancy trapping energy to $\Delta g_{v}^{0}=-0.5 \mathrm{eV}$ or below 
would prevent this loss of confinement: The oxygen vacancies mainly redistribute to the interface, they are strongly depleted close to the interface, and the ambipolar diffusion becomes negligible. In the depletion layer, the electron concentration is large, while the oxygen vacancy concentration is so low that the low-temperature electron mobility is only limited by scattering at the background concentration of impurities. Thus, a region of high conductivity develops on the first nanometers of the depletion layer that remains stable over many years. Consequently, engineering the interface to achieve such an oxygen vacancy trapping energy might create a strong and stable electron-defect separation, which enables a highly conductive and confined $\mathrm{SrTiO}_{3}$-based 2D electron system.

\section{ACKNOWLEDGMENTS}

D.V.C. acknowledges support by the NICE project, which has received funding from the Independent Research Fund Denmark, Grant No. 6111-00145B. F.G. acknowledges financial support from DFG FG 1604 (No. 315025796) and from the European Unions Horizon 2020 research and innovation program under Marie Sklodowska-Curie Grant Agreement No. 713683 (COFUNDfellowsDTU).
[1] N. Reyren, S. Thiel, A. D. Caviglia, L. F. Kourkoutis, G. Hammerl, C. Richter, C. W. Schneider, T. Kopp, A.-S. Ruetschi, D. Jaccard, M. Gabay, D. A. Muller, J.-M. Triscone, and J. Mannhart, Science 317, 1196 (2007).

[2] A. D. Caviglia, S. Gariglio, N. Reyren, D. Jaccard, T. Schneider, M. Gabay, S. Thiel, G. Hammerl, J. Mannhart, and J.-M. Triscone, Nature (London) 456, 624 (2008).

[3] S. Gariglio, A. Fête, and J. Triscone, J. Phys.: Condens. Matter 27, 283201 (2015).

[4] S. Okamoto and A. J. Millis, Nature (London) 428, 630 (2004).

[5] A. Brinkman, M. Huijben, M. Van Zalk, J. Huijben, U. Zeitler, J. C. Maan, W. G. Van der Wiel, G. Rijnders, D. H. A. Blank, and H. Hilgenkamp, Nat. Mater. 6, 493 (2007).

[6] L. Li, C. Richter, J. Mannhart, and R. C. Ashoori, Nat. Phys. 7, $762(2011)$

[7] J.-S. Lee, Y. W. Xie, H. K. Sato, C. Bell, Y. Hikita, H. Y. Hwang, and C.-C. Kao, Nat. Mater. 12, 703 (2013).

[8] M. Salluzzo, S. Gariglio, D. Stornaiuolo, V. Sessi, S. Rusponi, C. Piamonteze, G. M. De Luca, M. Minola, D. Marre, A. Gadaleta, H. Brune, F. Nolting, N. B. Brookes, and G. Ghiringhelli, Phys. Rev. Lett. 111, 087204 (2013).

[9] F. Gunkel, C. Bell, H. Inoue, B. Kim, A. G. Swartz, T. A. Merz, Y. Hikita, S. Harashima, H. K. Sato, M. Minohara, S. Hoffmann-Eifert, R. Dittmann, and H. Y. Hwang, Phys. Rev. X 6, 031035 (2016).

[10] D. V. Christensen, Y. Frenkel, Y. Chen, Y. Xie, Z. Chen, Y. Hikita, A. Smith, L. Klein, H. Hwang, N. Pryds, and B. Kalisky, Nat. Phys. 15, 269 (2019).

[11] H. Ohta, S. Kim, Y. Mune, T. Mizoguchi, K. Nomura, S. Ohta, T. Nomura, Y. Nakanishi, Y. Ikuhara, M. Hirano, H. Hosono, and K. Koumoto, Nat. Mater. 6, 129 (2007).

[12] A. Ruegg, S. Pilgram, and M. Sigrist, Phys. Rev. B 77, 245118 (2008).

[13] J. Mannhart and D. Schlom, Science 327, 1607 (2010).

[14] S. Thiel, G. Hammerl, A. Schmehl, C. W. Schneider, and J. Mannhart, Science 313, 1942 (2006).

[15] D. V. Christensen, F. Trier, M. von Soosten, G. Prawiroatmodjo, T. Jespersen, Y. Chen, and N. Pryds, Appl. Phys. Lett. 109, 021602 (2016).

[16] J. Delahaye and T. Grenet, J. Phys. D: Appl. Phys. 49, 395303 (2016).

[17] W. Niu, Y. Zhang, Y. Gan, D. V. Christensen, M. V. Soosten, E. J. Garcia-Suarez, A. Riisager, X. Wang, Y. Xu, R. Zhang, N. Pryds, and Y. Chen, Nano Lett. 17, 6878 (2017).
[18] A. Fète, C. Cancellieri, D. Li, D. Stornaiuolo, A. D. Caviglia, S. Gariglio, and J. Triscone, Appl. Phys. Lett. 106, 051604 (2015).

[19] C. Xu, C. Baeumer, R. A. Heinen, S. Hoffmann-Eifert, F. Gunkel, and R. Dittmann, Sci. Rep. 6, 22410 (2016).

[20] F. Gunkel, C. Lenser, C. Baeumer, F. Borgatti, F. Offi, G Panaccione, and R. Dittmann, APL Mater. 6, 076104 (2018).

[21] F. V. E. Hensling, D. J. Keeble, J. Zhu, S. Brose, C. Xu, F. Gunkel, S. Danylyuk, S. S. Nonnenmann, W. Egger, and R. Dittmann, Sci. Rep. 8, 8846 (2018).

[22] F. V. Hensling, C. Baeumer, M. Rose, F. Gunkel, and R. Dittmann, Mater. Res. Lett. 8, 31 (2020).

[23] D. V. Christensen, M. von Soosten, F. Trier, T. S. Jespersen, A. Smith, Y. Chen, and N. Pryds, Adv. Electron. Mater. 3, 1700026 (2017).

[24] Y. Xie, C. Bell, Y. Hikita, S. Harashima, and H. Y. Hwang, Adv. Mater. 25, 4735 (2013).

[25] K. Han, K. Hu, X. Li, K. Huang, Z. Huang, S. Zeng, D. Qi, C. Ye, J. Yang, H. Xu, A. Ariando, J. Yi, W. Lu, S. Yan, and X. R. Wang, Sci. Adv. 5, eaaw7286 (2019).

[26] F. Gunkel, P. Brinks, S. Hoffmann-Eifert, R. Dittmann, M Huijben, J. E. Kleibeuker, G. Koster, G. Rijnders, and R. Waser, Appl. Phys. Lett. 100, 052103 (2012).

[27] F. Gunkel, R. Waser, A. H. H. Ramadan, R. A. De Souza, S. Hoffmann-Eifert, and R. Dittmann, Phys. Rev. B 93, 245431 (2016).

[28] A. Ohtomo and H. Y. Hwang, Nature (London) 427, 423 (2004).

[29] N. Nakagawa, H. Y. Hwang, and D. A. Muller, Nat. Mater. 5, 204 (2006).

[30] D. V. Christensen and A. Smith, Appl. Surf. Sci. 423, 887 (2017).

[31] G. Herranz, M. Basletic, M. Bibes, C. Carretero, E. Tafra, E. Jacquet, K. Bouzehouane, C. Deranlot, A. Hamzic, J. M. Broto, A. Barthelemy, and A. Fert, Phys. Rev. Lett. 98, 216803 (2007).

[32] A. Kalabukhov, R. Gunnarsson, J. Borjesson, E. Olsson, T. Claeson, and D. Winkler, Phys. Rev. B 75, 121404(R) (2007).

[33] M. Basletic, J. Maurice, C. Carretero, G. Herranz, O. Copie, M. Bibes, E. Jacquet, K. Bouzehouane, S. Fusil, and A. Barthelemy, Nat. Mater. 7, 621 (2008).

[34] A. F. Santander-Syro, O. Copie, T. Kondo, F. Fortuna, S Pailhes, R. Weht, X. G. Qiu, F. Bertran, A. Nicolaou, A. Taleb-Ibrahimi, P. Le Fevre, G. Herranz, M. Bibes, N. Reyren, Y. Apertet, P. Lecoeur, A. Barthelemy, and M. J. Rozenberg, Nature (London) 469, 189 (2011). 
[35] W. Meevasana, P. King, R. He, S. Mo, M. Hashimoto, A. Tamai, P. Songsiriritthigul, F. Baumberger, and Z. Shen, Nat. Mater. 10, 114 (2011).

[36] S. McKeown Walker, A. de la Torre, F. Y. Bruno, A. Tamai, T. K. Kim, M. Hoesch, M. Shi, M. S. Bahramy, P. D. C. King, and F. Baumberger, Phys. Rev. Lett. 113, 177601 (2014).

[37] L. Dudy, M. Sing, P. Scheiderer, J. D. Denlinger, P. Schütz, J. Gabel, M. Buchwald, C. Schlueter, T.-L. Lee, and R. Claessen, Adv. Mater. 28, 7443 (2016).

[38] S. Gonzalez, C. Mathieu, O. Copie, V. Feyer, C. M. Schneider, and N. Barrett, Appl. Phys. Lett. 111, 181601 (2017).

[39] A. Chikina, M. Caputo, M. Naamneh, D. V. Christensen, T. Schmitt, M. Radovic, and V. N. Strocov, Adv. Funct. Mater. 29, 1900645 (2019).

[40] F. Y. Bruno, J. Tornos, M. Gutierrez del Olmo, G. Sanchez Santolino, N. M. Nemes, M. Garcia-Hernandez, B. Mendez, J. Piqueras, G. Antorrena, L. Morellon, J. M. De Teresa, M. Clement, E. Iborra, C. Leon, and J. Santamaria, Phys. Rev. B 83, 245120 (2011).

[41] Y. Chen, N. Pryds, J. E. Kleibeuker, G. Koster, J. Sun, E. Stamate, B. Shen, G. Rijnders, and S. Linderoth, Nano Lett. 11, 3774 (2011).

[42] Y. Z. Chen, N. Bovet, F. Trier, D. V. Christensen, F. M. Qu, N. H. Andersen, T. Kasama, W. Zhang, R. Giraud, J. Dufouleur, T. S. Jespersen, J. R. Sun, A. Smith, J. Nygard, L. Lu, B. Buechner, B. G. Shen, S. Linderoth, and N. Pryds, Nat. Commun. 4, 1371 (2013).

[43] F. Gunkel, S. Hoffmann-Eifert, R. A. Heinen, D. V. Christensen, Y. Z. Chen, N. Pryds, R. Waser, and R. Dittmann, ACS Appl. Mater. Interfaces 9, 1086 (2017).

[44] A. Ohtomo and H. Y. Hwang, J. Appl. Phys. 102, 083704 (2007).

[45] R. A. De Souza, Phys. Chem. Chem. Phys. 11, 9939 (2009).

[46] R. A. De Souza, F. Gunkel, S. Hoffmann-Eifert, and R. Dittmann, Phys. Rev. B 89, 241401(R) (2014).

[47] P. Schutz, D. V. Christensen, V. Borisov, F. Pfaff, P. Scheiderer, L. Dudy, M. Zapf, J. Gabel, Y. Z. Chen, N. Pryds, V. A. Rogalev, V. N. Strocov, C. Schlueter, T.-L. Lee, H. O. Jeschke, R. Valenti, M. Sing, and R. Claessen, Phys. Rev. B 96, 161409(R) (2017).

[48] P. Schutz, F. Pfaff, P. Scheiderer, Y. Z. Chen, N. Pryds, M. Gorgoi, M. Sing, and R. Claessen, Phys. Rev. B 91, 165118 (2015).

[49] M. Yazdi-Rizi, P. Marsik, B. P. Mallett, A. Dubroka, D. V. Christensen, Y. Chen, N. Pryds, and C. Bernhard, EPL 113, 47005 (2016).

[50] M. Huijben, G. Koster, M. K. Kruize, S. Wenderich, J. Verbeeck, S. Bals, E. Slooten, B. Shi, H. J. Molegraaf, J. E. Kleibeuker, S. van Aert, J. B. Goedkoop, A. Brinkman, D. H. A. Blank, M. S. Golden, G. van Tendeloo et al., Adv. Funct. Mater. 23, 5240 (2013).

[51] Y. Z. Chen, F. Trier, T. Wijnands, R. J. Green, N. Gauquelin, R. Egoavil, D. V. Christensen, G. Koster, M. Huijben, N. Bovet, S. Macke, F. He, R. Sutarto, N. H. Andersen, J. A. Sulpizio, M. Honig, G. E. D. K. Prawiroatmodjo, T. S. Jespersen, S. Linderoth, S. Ilani, J. Verbeeck, G. Van Tendeloo, G. Rijnders, G. A. Sawatzky, and N. Pryds, Nat. Mater. 14, 801 (2015).

[52] Z. Wang, Z. Zhong, X. Hao, S. Gerhold, B. Stoeger, M. Schmid, J. Sanchez-Barriga, A. Varykhalov, C. Franchini, K.
Held, and U. Diebold, Proc. Natl. Acad. Sci. USA 111, 3933 (2014).

[53] D. V. Christensen, F. Trier, W. Niu, Y. Gan, Y. Zhang, T. S. Jespersen, Y. Chen, and N. Pryds, Adv. Mater. Interfaces 6, 1900772 (2019).

[54] M. von Soosten, D. V. Christensen, C.-B. Eom, T. S. Jespersen, Y. Chen, and N. Pryds, Sci. Rep. 9, 18005 (2019).

[55] N. Chan, R. Sharma, and D. Smyth, J. Electrochem. Soc. 128, 1762 (1981).

[56] R. Waser, Z. Naturforsch., A: Phys., Phys. Chem., Kosmophys. 42, 1357 (1987).

[57] R. Moos and K. H. Härdtl, J. Am. Ceram. Soc. 78, 2569 (1995).

[58] R. Meyer, R. Waser, J. Helmbold, and G. Borchardt, Phys. Rev. Lett. 90, 105901 (2003).

[59] K. Gömann, G. Borchardt, M. Schulz, A. Gömann, W. MausFriedrichs, B. Lesage, O. Kaïtasov, S. Hoffmann-Eifert, and T. Schneller, Phys. Chem. Chem. Phys. 7, 2053 (2005).

[60] F. A. Kröger and H. J. Vink, J. Phys. Chem. Solids, UK 5, 208 (1958).

[61] D. V. Christensen, Y. Frenkel, P. Schutz, F. Trier, S. Wissberg, R. Claessen, B. Kalisky, A. Smith, Y. Z. Chen, and N. Pryds, Phys. Rev. Appl. 9, 054004 (2018).

[62] D. Bednarczyk and J. Bednarczyk, Phys. Lett. A 64, 409 (1978).

[63] O. Copie, V. Garcia, C. Bodefeld, C. Carretero, M. Bibes, G. Herranz, E. Jacquet, J. L. Maurice, B. Vinter, S. Fusil, K. Bouzehouane, H. Jaffres, and A. Barthelemy, Phys. Rev. Lett. 102, 216804 (2009).

[64] M. Minohara, Y. Hikita, C. Bell, H. Inoue, M. Hosoda, H. Sato, H. Kumigashira, M. Oshima, E. Ikenaga, and H. Hwang, Sci. Rep. 7, 9516 (2017).

[65] J. Hemberger, P. Lunkenheimer, R. Viana, R. Bohmer, and A. Loidl, Phys. Rev. B 52, 13159 (1995).

[66] C. Wagner, Z. Phys. Chem. Abt. B 21, 25 (1933).

[67] F. D. Gealy and H. L. Tuller, J. Phys. Colloq. 51, C1-483 (1990)

[68] R. Waser, J. Am. Ceram. Soc. 74, 1934 (1991).

[69] M. Schie, R. Waser, and R. A. De Souza, J. Phys. Chem. C 118, 15185 (2014).

[70] R. A. De Souza, Adv. Funct. Mater. 25, 6326 (2015).

[71] H. Schraknepper, T. E. Weirich, and R. A. De Souza, Phys. Chem. Chem. Phys. 20, 15455 (2018).

[72] R. Moos and K. H. Härdtl, J. Am. Ceram. Soc. 80, 2549 (1997).

[73] O. N. Tufte and P. W. Chapman, Phys. Rev. 155, 796 (1967).

[74] H. P. R. Frederikse and W. Hosler, Phys. Rev. 161, 822 (1967).

[75] R. Moos, W. Menesklou, and K. H. Härdtl, Appl. Phys. A 61, 389 (1995).

[76] R. Moos and K. H. Härdtl, J. Appl. Phys. 80, 393 (1996).

[77] F. Trier, D. V. Christensen, and N. Pryds, J. Phys. D: Appl. Phys. 51, 293002 (2018).

[78] A. D. Caviglia, S. Gariglio, C. Cancellieri, B. Sacépé, A. Fète, N. Reyren, M. Gabay, A. F. Morpurgo, and J.-M. Triscone, Phys. Rev. Lett. 105, 236802 (2010).

[79] See Supplemental Material at http://link.aps.org/supplemental/ 10.1103/PhysRevMaterials.4.104604 for detailed information on the influence of the initial length scale of the oxygen vacancy profile, the used approximation of the Fermi integral $F_{1 / 2}\left(\eta_{n}\right)$, the calculation of the lookup table for $\varepsilon_{r}(T, E)$, the simulated redistribution of oxygen vacancies without an oxygen vacancy trapping energy, and the occurrence of the local minimum in the electron profiles at low temperatures. 